\title{
Parabronchial angioarchitecture in developing and adult chickens
}

\author{
A. N. Makanya ${ }^{1,2}$ and V. Djonov ${ }^{1}$ \\ ${ }^{1}$ Institute of Anatomy, Department of Medicine, Fribourg University, Fribourg, Switzerland; and ${ }^{2}$ Department of Veterinary \\ Anatomy and Physiology, University of Nairobi, Nairobi, Kenya
}

\begin{abstract}
Makanya AN, Djonov V. Parabronchial angioarchitecture in developing and adult chickens. J Appl Physiol 106: 1959-1969, 2009. First published March 26, 2009; doi:10.1152/japplphysiol.91570.2008.—The avian lung has a highly sophisticated morphology with a complex vascular system. Extant data regarding avian pulmonary angioarchitecture are few and contradictory. We used corrosion casting techniques, light microscopy, as well as scanning and transmission electron microscopy to study the development, topography, and distribution of the parabronchial vasculature in the chicken lung. The arterial system was divisible into three hierarchical generations, all formed external to the parabronchial capillary meshwork. These included the interparabronchial arteries (A1) that ran parallel to the long axes of parabronchi and gave rise to orthogonal parabronchial arteries (A2) that formed arterioles (A3). The arterioles formed capillaries that participated in the formation of the parabronchial mantle. The venous system comprised six hierarchical generations originating from the luminal aspect of the parabronchi, where capillaries converged to form occasional tiny infundibular venules (V6) around infundibulae, or septal venules (V5) between conterminous atria. The confluence of the latter venules formed atrial veins (V4), which gave rise to intraparabronchial veins (V3) that traversed the capillary meshwork to join the interparabronchial veins (V1) directly or via parabronchial veins (V2). The primitive networks inaugurated through sprouting, migration, and fusion of vessels and the basic vascular pattern was already established by the 20th embryonic day, with the arterial system preceding the venous system. Segregation and remodeling of the fine vascular entities occurred through intussusceptive angiogenesis, a process that probably progressed well into the posthatch period. Apposition of endothelial cells to the attenuating epithelial cells of the air capillaries resulted in establishment of the thin blood-gas barrier. Fusion of blood capillaries proceeded through apposition of the anastomosing sprouts, with subsequent thinning of the abutting boundaries and ultimate communication of the lumens. Orthogonal reorientation of the blood capillaries at the air capillary level resulted in a cross-current system at the gas exchange interface.
\end{abstract}

chick development; pulmonary angioarchitecture; intussusceptive angiogenesis; sprouting angiogenesis

THE AVIAN LUNG HAS BEEN NOTED to be complex, both in structure and function (11). Recently, we have documented the precise arrangement of the air conduits in the lung of the domestic fowl with the notion that the anatomy of the latter structures has been misrepresented over the years (22). Generally the functional design of the avian respiratory system remains recondite, despite concerted efforts to unravel the mysteries of its architecture (18). As we have indicated recently, recruitment of several techniques may be requisite in elucidating the structural and topographical details of the avian lung (22), and studying of the structures during the developmental process has an overt, added advantage $(12,22)$.

Address for reprint requests and other correspondence: V. G. Djonov, Institute of Anatomy, Dept. of Medicine, Fribourg Univ., Rte. Albert Gockel 1, CH-1700 Fribourg, Switzerland (e-mail: valentin.djonov@unifr.ch).
Detailed accounts on the organization of pulmonary circulation in the avian lung preponderate in literature $(1,14,15$, 34). The functional supply to the lung is through the pulmonary artery, which enters the lung at the hilus and breaks into a cranial, caudomedial, and a caudolateral artery. These latter vessels give rise to several branches that form the interparabronchial arteries (1). Septal venules occur in the interatrial septa and drain adjacent atria, while atrial veins drain circumscribed regions of the parabronchus and join the intraparabronchial veins. Intraparabronchial veins traverse the parabronchial exchange tissue radially to join interparabronchial veins, which, in turn, contribute to the cranial and caudal radices of the pulmonary vein $(1,7,11,24)$. While there appears to be a consensus on the disposition of these large vessels, the pattern at the parabronchial level is less overt, and details in literature provide conflicting information. Several reports indicate that the interparabronchial arteries run parallel and also transverse to the long axes of the parabronchi $(1,11,20)$. In contrast, Duncker (8) indicated that the blood capillaries of the exchange tissue originate only from the periphery of the parabronchus, while other authors allege that intraparabronchial arteries and arterioles penetrate the exchange tissue to form the anastomosing networks of capillaries $(28,34)$. These disparate observations may be attributed to the limitations of the investigative techniques employed and failure to follow the inauguration of structures during their formative stages, a strategy belabored by previous authors $(12,22)$.

The rigidity of the avian lung obtains from the intricate three-dimensional (3D) arrangement of the air conduits and blood vessels, so that these structures support one another (17, 33). Distensibility of the avian pulmonary capillaries in the face of increased pressure in the lung has been shown to be only $13 \%$, compared with $128 \%$ attained in the compliant mammalian lung (32). During embryogenesis, it has been demonstrated that pulmonary vasculature development is driven by vasculogenic (3), as well as angiogenic (23), mechanisms. Development of the air conduits precedes and guides the patterning of the developing vasculature, and both sprouting and intussusceptive angiogenic mechanisms remodel the establishment of the final vascular patterns (23). The topographical arrangement of the parabronchial vasculature has been presented by West et al. (34) and King and McLelland (11). However, details on the finer generations of the vessels and how such patterns are accomplished from the latter two studies are either missing or speculative. Our recent study on avian pulmonary angiogenesis (27) highlighted the mechanisms through which the fine capillary meshwork is established, but the patterning of the larger vessels and the accomplishment of structure-specific angioarchitecture were not addressed.

In the present study, we have used microscopic techniques to study the development and definitive topographical arrange- 
Table 1. Age, study techniques, and number of specimens used for each technique

\begin{tabular}{clcc}
\hline \hline Embryonic Age & \multicolumn{1}{c}{ Techniques Applied } & $\begin{array}{c}\text { Number of } \\
\text { Specimens }\end{array}$ & $\begin{array}{c}\text { Total Number } \\
\text { of Specimens }\end{array}$ \\
\hline E13 & Semithin & 5 & 5 \\
E15 & Combined intravascular and & 4 & 4 \\
& $\quad$ intratracheal casting \\
E16 & Semithin sections & 5 & 5 \\
E18 & Intravascular casting & 3 & 3 \\
& Semithin sections and TEM & 3 & 3 \\
E19 & TEM & 5 & 5 \\
E20 & Intravascular casting & 5 & 5 \\
E21 & Semithin sections & 5 & 5 \\
Adults & Intravascular casting & 3 & 3 \\
\hline
\end{tabular}

E, embryonic day; TEM, transmission electron microscopy.

ment of parabronchial vasculature in embryonic and adult birds. We present unambiguous data elucidating the 3D arrangement of the extant parabronchial blood vessels. The direction of blood flow in relation to air flow has also been documented while identifying the supplying and draining categories.

\section{MATERIAL AND METHODS}

Experimental animals. Fertilized Brown Leghorn eggs were incubated at $37^{\circ} \mathrm{C}$ and a humidity of $65 \%$. Embryos covering Hamburger and Hamilton (HH) stages 38-46 [embryonic days 13-21 (E13-E21)] were obtained and processed, as detailed below. In addition, adult Rhode Island Red layers (Gallus gallus variant domesticus), already identified for culling, were procured for the study. For late-stage embryos from E18 and adult birds, anesthesia was achieved by intra-abdominal injection of Euthatal (sodium pentobarbitone). Specimens were chosen to represent early, middle, and late stages of vessel pattern formation, as well the ultimate disposition in adults.

The numbers and ages of specimens used for various techniques are presented in Table 1. All protocols were approved by the Animal Care and Use Committee of the University of Nairobi.

Light and electron microscopy. Specimens for both light microscopy and electron microscopic studies were fixed using a solution of $2.5 \%$ glutaraldehyde in $0.1 \mathrm{M}$ cacodylate buffer ( $\mathrm{pH} \mathrm{7.4,350} \mathrm{mosmol/}$ $\mathrm{kgH}_{2} \mathrm{O}$ ). Embryos below 5 days of incubation were fixed by total immersion in the fixative, while the rest were fixed by perfusion through the right ventricle. Lungs were dissected out and diced into small blocks. Tissue blocks were postfixed in osmium tetroxide, block stained using uranyl acetate, dehydrated through ascending concentrations of ethanol, and embedded in epoxy resin. Semithin sections were obtained at a nominal thickness of $1 \mu \mathrm{m}$, stained with toluidine blue, and viewed under a Coolscope Digital Microscope (Nikon). Ultrathin sections were obtained at $90 \mathrm{~nm}$, counterstained with lead citrate, and viewed on a Philips EM-300 microscope.

Intravascular casting. Lungs from adult birds and E13-E21 (HH stages 38-46) embryos were used for microscopic observations of the parabronchial vessels. Embryos were cultured shell free in petri dishes, and appropriate HH stages were selected for vascular casting. The embryos were perfused with a solution of $0.9 \%$ sodium chloride containing $1 \%$ Liqemin and $1 \%$ procaine through the vitelline artery. The vasculature was then filled with methylmethacrylate resin (Mercox, Vilene Hospital, Tokyo, Japan) containing $0.1 \mathrm{ml}$ accelerator per every $5 \mathrm{ml}$ of the resin or with Technovit 3040 powder mixed with catalyst in the ratio $1: 1$, as per the manufacturer's instructions. One hour after perfusion, either the entire embryo or specific targeted organs were immersed in Ringer solution for at least $2 \mathrm{~h}$ and subsequently transferred to a $15 \%$ potassium hydroxide solution for 2-4 wk. After dissolution of the tissues, the embryos and organs were washed, dehydrated in ascending concentrations of ethanol, and dried in a desiccator. Samples were mounted on aluminium stubs, sputter coated with gold, and viewed under a Philips XL 30 FEG scanning electron microscope. For the adult birds, perfusion was done either through the pulmonary artery or through the right ventricle of the heart, and then the lungs were filled with methyl methacrylate resin, dissected out, and processed for scanning electron microscopy, as described above.

Double casting. The relationship between the developing air conduits and the incipient vasculature was captured in double casting (i.e., combined intravascular and intratracheal casting) preparations. Methylmethacrylate resin was introduced first through left ventricle and then through the tracheae of selected embryos. The entire pulmonary vasculature was filled with methylmethacrylate resin or with Technovit 3040 powder and then processed further for the scanning electron microscope, as detailed above.

\section{RESULTS}

The pattern of blood vessels associated with the parabronchi was recognizable as an arterial system with three vascular generations (A1, A2, and A3), located external to the parabronchial capillary meshwork, and a venous system with six generations of veins (V1-V6). The veins were located either internal, within, or external to the capillary mantle. Precise arrangement of such vessels was captured by intravascular corrosion casting in late-stage embryos and in adult lungs (Fig. 1), as well as in semithin sections of the embryonic lung (Fig. 2). The parabronchial capillary meshwork was formed into hexagonal "cylinders" packed tightly and separated by a thin layer of connective tissue, known as the interparabronchial septum (Fig. 2). It is this septum that contained the interparabronchial

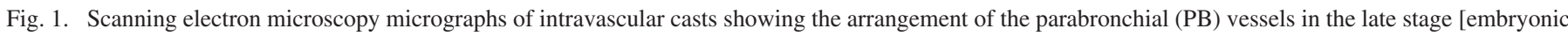

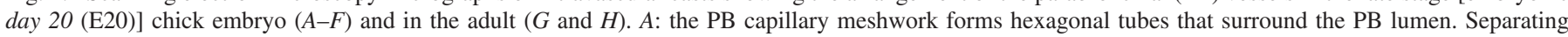

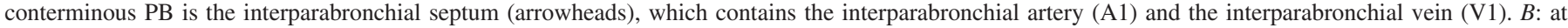

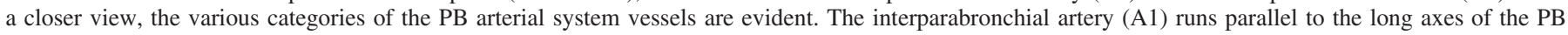

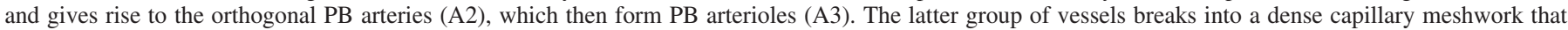

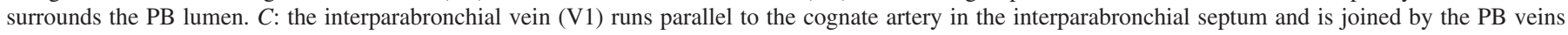

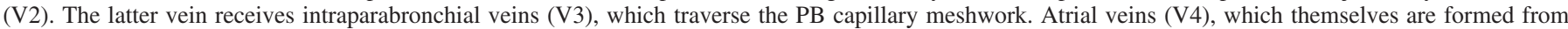

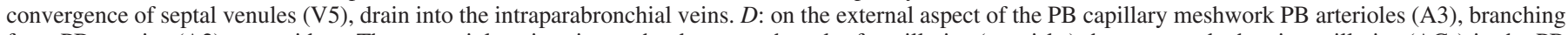

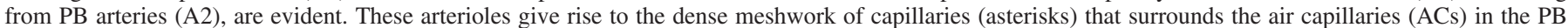

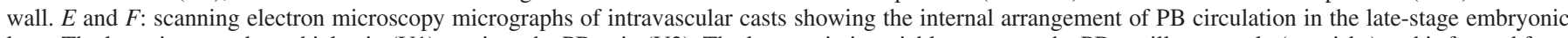

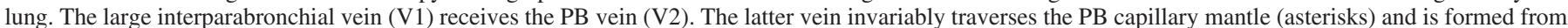

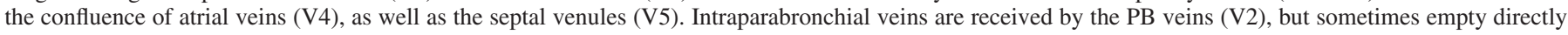

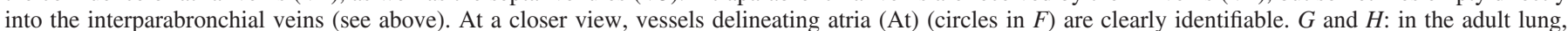

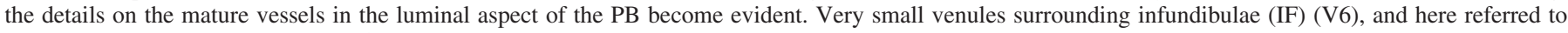

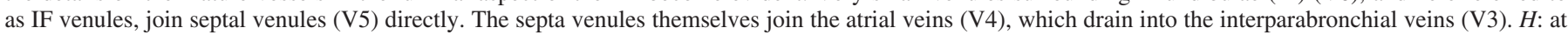

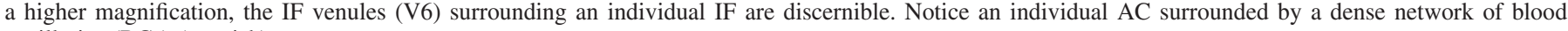
capillaries (BCs) (asterisk). 

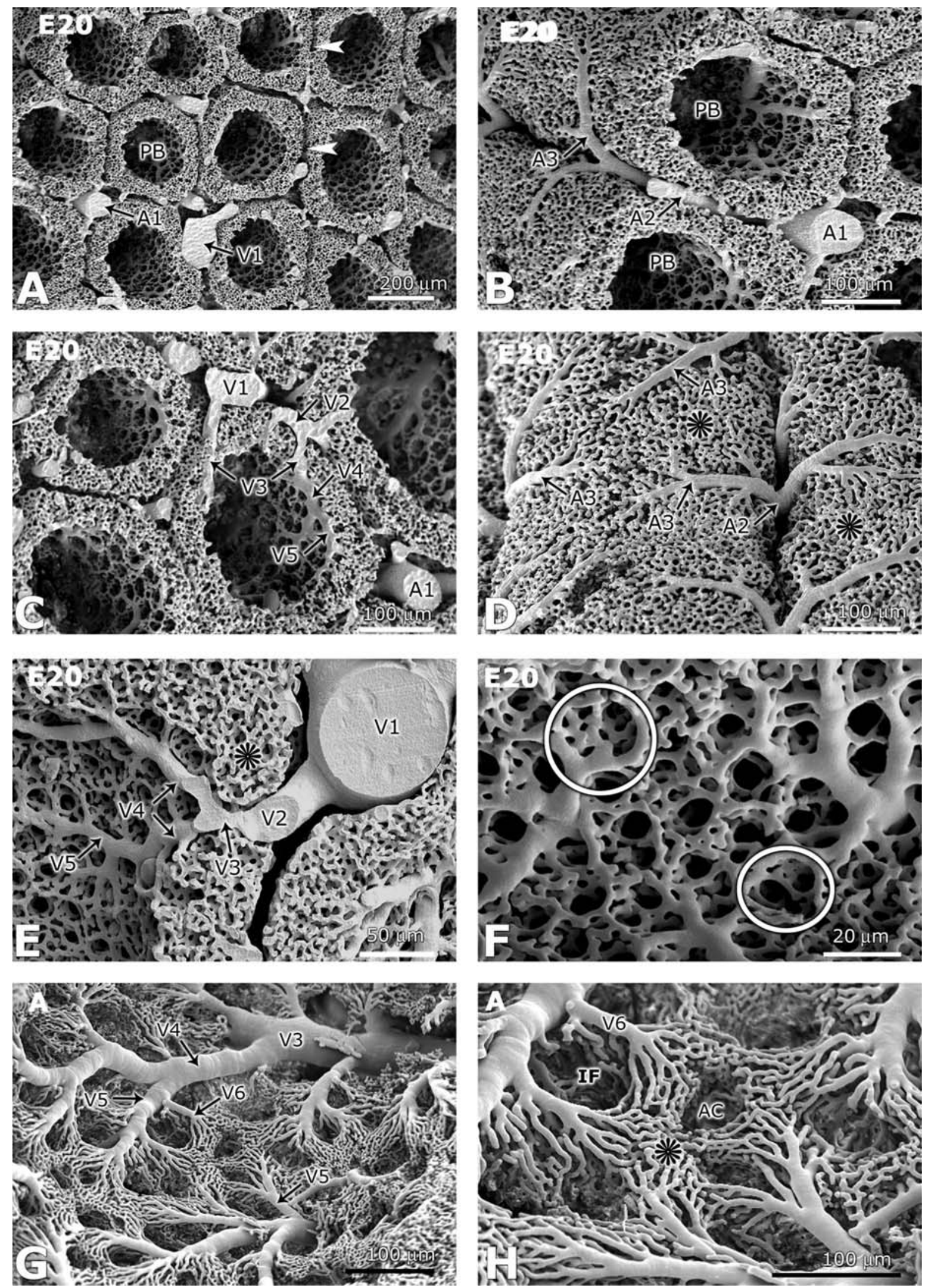

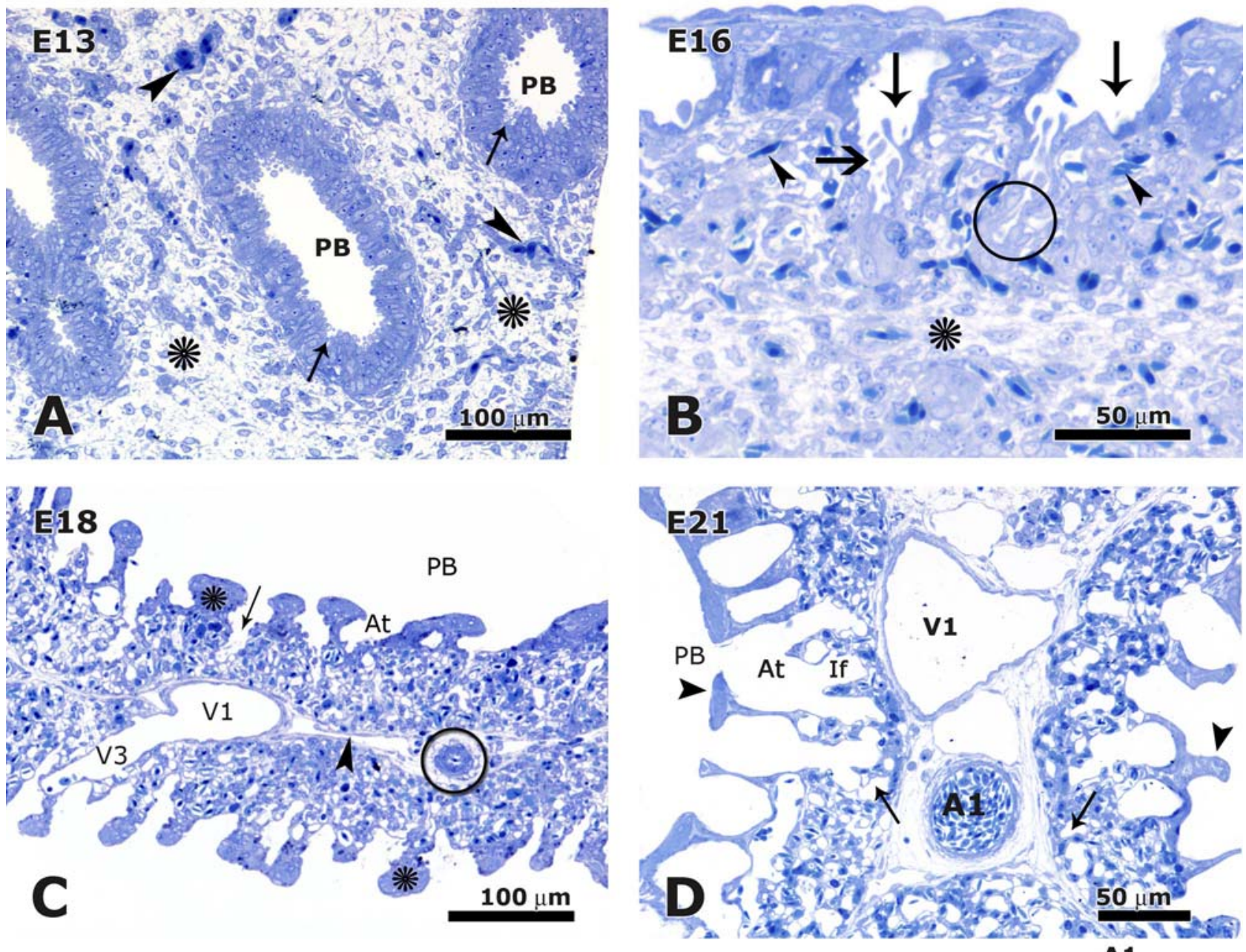

A1

Fig. 2. Semithin sections showing the establishment of the PB blood vessels. A: by E13, the PB is the characteristic structure in the lung parenchyma. The tissue of the interparabronchial septum (asterisks) at this stage is abundant, and a few vascular profiles (arrowheads), as well as formative At (arrows), are encountered. $B$ : the formative At (vertical arrows) start to grow toward the mesenchymal tissue (asterisks) of the septum by E16. The interparabronchial septum is clearly delineated, and vascular profiles (arrowheads) of the incipient PB vessels are evident. The formative IF is indicated with a horizontal arrow, while the circle indicates a developing AC. C: by E18, the PB parenchymal tissue has replaced most of the mesenchyme, so that only a thin interparabronchial septum is evident (arrowhead). The interparabronchial vessels include the interparabronchial vein (V1), the intraparabronchial vein (V3), and interparabronchial artery (encircled). The intraparabronchial vein traverses the PB wall to join the atrial capillary network. Note the interatrial septa (asterisks) projecting into the PB lumina. An At and an incipient IF (arrow) are also shown. D: the PB parenchymal tissue at E21 closely resembles that of the adult chicken. Gas exchange parenchyma has replaced the mesenchyme, so that only the interparabronchial vessels stand out prominently. These include the interparabronchial vein (V1) and the intraparabronchial artery (A1). Note the interatrial septa (arrowheads) projecting into the PB lumina. The At give rise to IF, which form ACs (arrow).

vessels, which included the interparabronchial artery (A1) and the interparabronchial vein (V1) (Figs. 1, $A-D$, and 2). The interparabronchial artery ran parallel to the long axes of the parabronchus in the interparabronchial septum and gave rise to orthogonal parabronchial arteries (A2), which, in turn, formed parabronchial arterioles (A3) (Fig. 1, $B$ and $D$ ). These arterioles broke into a dense network of capillaries that surrounded the atria and infundibulae and also penetrated deeper into the meshwork to interlace with air capillaries, finally converging at the internal aspect of the parabronchus to inaugurate the venous system (Fig. 1, $E-H$ ). Notably, the ultimate general direction of flow in the blood capillaries in the adult lung was perpendicular to that of the air capillaries, thus establishing a cross-current system.

The interparabronchial vein (V1) ran alongside the cognate artery in the interparabronchial septum and received several parabronchial veins. The parabronchial veins were formed by convergence of intraparabronchial veins (V3), which traversed the parabronchial capillary meshwork and emanated from the interior aspect of the parabronchial capillary mantle. The intraparabronchial veins were formed from atrial veins (V4), which themselves resulted from convergence of septal venules (V5) (Fig. 1, $C, E$, and $G$ ). The septal venules formed directly from the parabronchial capillaries and from convergence of infundibular venules and were located in the septa between conterminous atria. Occasionally, capillaries converged around an infundibulum to form small venules (V6), referred to here as infundibular venules (Fig. 1, $G$ and $H$ ).

In the developing lung at E13, no interparabronchial vessels were evident, and only a few vascular profiles were discernible. Atria had not formed, so that the interparabronchial septum was thick and laden with many undifferentiated cells (Fig. 2A). 
The septum, however, by E15, was much thinner and was invaded by formative atria and infundibulae, and some interparabronchial vessels were evident (Fig. 2B). By E18, the phenotype of the parabronchial unit was definite, with a thin septum containing veins and arteries and exchange tissue with atria, infundibulate, and air and blood capillaries (Fig. 2, $C$ and $D)$. As shown in Table 1, the flow direction in relation to the air varies with the generation of the blood vessel, being cocurrent at the interparabronchial artery (A1), countercurrent at the interparabronchial vein (V1), and cross-current at the blood capillary level.

The progress of vascular development was followed from E15, where, evidently, the large air conduits, which included the primary and secondary bronchi, were well formed ahead of the parabronchial capillary mantle and guided the formation of the vascular network. Treelike tufts of incipient capillaries were visible around the air conduits (Fig. 3A), and the early incipient atria appeared like small mounds on the secondary bronchi (Fig. 3B). The parabronchial capillary meshwork was better developed by E18, so that the parabronchial lumens were surrounded by the capillary mantle, and the interparabronchial vessels were clearly identifiable (Fig. 3C). The parabronchial meshwork, however, was less geometrical compared with the late-stage embryos and was formed into rounded or ovoid rather than hexagonal profiles (Fig. 3C). On the external surface of the parabronchial capillary mantle, it was clear that the migrating arterioles approached one another and fused at the level of the long axis of the parabronchus, and their continuation was broken down to become part of the capillary meshwork (Fig. 3, D, E, and $F$ ).

The establishment of the venous system on the luminal aspect of the parabronchi and the exchange capillaries was achieved by both sprouting and intussusceptive angiogenesis (Fig. 4). Initially, migrating vascular entities approximated each other around the developing air conduits and fused. This resulted in delineation of the various generations of the venous system, which included the septal venules (V5), atrial veins (V4), as well as the intraparabronchial veins (V3) (Fig. 4). Subsequently, intussusceptive angiogenesis resulted in delineation of finer vascular branches (Fig. 4, C and D), which became the exchange capillaries. The delineated incipient capillaries grew toward the parabronchial wall and hence participated in the establishment of the dense capillary meshwork (Fig. 4E). The various generations of the venous system were easily identifiable at E18, even before the processes of vascular anastomoses were complete (Fig. 4E). Intussusceptive vascular remodeling finally resulted in fine smooth vascular entities encountered in the adult chicken lung (Fig. 4, $G$ and $H$ ).

The interaction between the developing blood capillaries and air capillaries and also the anastomoses of blood capillaries was captured at ultrastructural level (Fig. 5). The newly formed blood capillaries were essentially thin walled, while the air capillary epithelia underwent complex processes of secarecytosis that progressively cut the cells to a low level, suitable for formation of the blood-gas barrier. These processes included vacuolation and vesiculation (Fig. 5, $A$ and $B$ ), and the air capillaries were easily distinguished from blood capillaries due to the presence of numerous vesicles, vacuoles, and apical microplicae (Fig. 5, $A$ and $B$ ). Blood capillary fusions appeared to proceed through progressive approximation of the anastomosing ends and subsequent thinning of the conterminous boundaries until a communication was established (Fig. 5, C and $D$ ). Ultimately, a close association between the air capillaries and the blood capillaries was established, whereby the blood capillaries had a general orthogonal orientation to the air capillaries (Fig. 6), thus establishing a cross-current system.

\section{DISCUSSION}

Confusion in the extant literature regarding certain details of the functional morphology of the avian lung has recently been highlighted (22). While the said discrepancies have affected the nomenclature and description of the air conduits, little information on the parabronchial vasculature exists. A detailed account of the avian pulmonary vasculature was reported by Abdalla and King (1, 2). The latter authors used several injection techniques to map out the blood vessels, but lacked the resolution furnished by the scanning electron microscope employed in the present study. Furthermore, use of developing embryos has availed details of the formative stages of the blood vessels, allowing scrutiny of their temporospatial arrangement.

The observations on the parabronchial circulation in the present study differ appreciably from what is reported in literature. Interparabronchial arteries (A1) actually form parabronchial arteries (A2), which break into parabronchial arterioles (A3). The latter two categories of vessels do not penetrate the parabronchial capillary mantle, and hence the names intraparabronchial artery $(11,19,34)$ and intraparabronchial arteriole $(11,15,20)$ do not appropriately describe the vessels. Conversely, the septal venules (V5) occurring in the interatrial septa receive blood from the parabronchial capillaries and drain into the atrial veins (V4). Several branches of the latter category of veins then join up to form the intraparabronchial veins (V3) that traverse the parabronchial capillary mantle to either join the parabronchial veins (V2) or empty directly into the interparabronchial veins (V1). The atrial veins and the intraparabronchial veins also receive blood from intraparabronchial venules (11), referred here also as infundibular venules, and capillary branches from the mantle and then continue either to parabronchial veins or directly to the interparabronchial veins. No other large blood vessels are found in the parabronchial capillary mantle. This is functionally significant, since absence of large vessels in the gas exchange mantle means that there is more space for interaction between air capillaries and blood capillaries, the interface where actual gas exchange takes place.

Notably, the convective air flow within the avian parabronchus is orthogonal to the blood flow at the level of parabronchials, and this probably has largely resulted in the system being designated cross-current $(9,31)$. Earlier confusion regarding whether the arrangement was countercurrent or crosscurrent (1) preponderated in literature. At the air capillary level, Nasu (28) reported possibility of both systems. In the present study, we find that the bulk of the blood capillaries in the parabronchial gas exchange mantle are perpendicular to the air capillaries, supporting a mainly cross-current system, although some capillaries change direction to a countercurrent disposition as they emerge toward the atrial openings (Fig. 6).

Gas exchange in the avian lung is described to follow a cross-current system, whereby the convective gas flow in the parabronchial lumen is orthogonal to the general flow of the blood in the exchange capillaries $(29,31)$. In such an arrangement, blood 

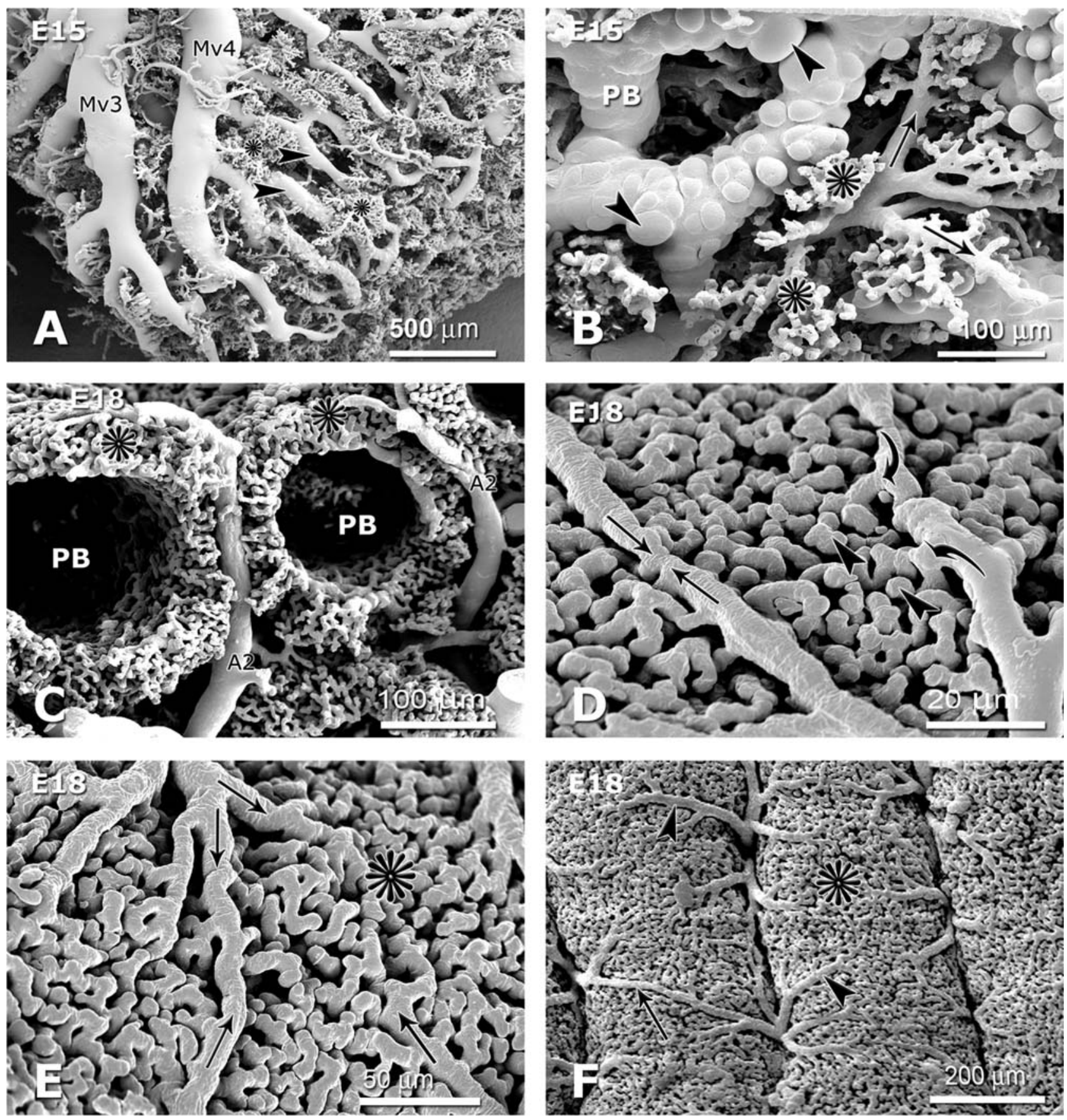

Fig. 3. Scanning electron micrographs of double (i.e., intravascular together with intratracheal; $A$ and $B$ ) and intravascular $(C-F)$ casts, showing the establishment of the PB capillary meshwork at various developmental stages. A: both the secondary bronchi [medioventrals 3 (MV3) and 4 (MV4)] and the PB (arrowheads) are well established, but only a few capillary tufts (asterisks) are present. These capillary tufts send branches to surround the formative PB (arrowheads) and the secondary bronchi. $B$ : a close-up showing the interaction between the developing vasculature and the formative At. The At (arrowheads) sprout from the PB ahead of the spreading capillaries (arrows), so that they guide the patterning. Note the capillary tufts (asterisks) sending branches that engulf the PB. $C$ : at E18, the primitive PB capillary mantle has already formed the typical cylindrical, albeit less geometrically refined, patterns (asterisks) surrounding the PB lumen. The interparabronchial vessels, such as the PB artery (A2), are clearly discernible. $D$ : on external aspect, the arterial system is represented by migrating arterioles that come to fuse with their opposite cognates (opposing arrows). Notice the capillary sprouts that establish the PB mantle (arrowheads). As soon as the arterioles abut, they start giving rise to capillary branches (curved arrows) that expand the capillary mantle toward the interior of the capillary mantle. $E$ : the processes of vascular remodeling happen very fast, so that the anastomosed arterioles (arrows) are broken down to form the dense capillary meshwork (asterisk) characteristic of the PB wall. F: vascular remodeling results in short PB arterioles (arrowheads) that feed the incipient capillary meshwork from the external aspect of the PB (asterisk). Notice a constricting remaining anastomosed arteriole (arrow).

entering a parabronchus at its origin equilibrates with the high $\mathrm{PO}_{2}$ and low $\mathrm{PCO}_{2}$ in the gas, while blood near the end of the parabronchus equilibrates with a gas that contains a much lower $\mathrm{PO}_{2}$ and higher $\mathrm{PCO}_{2}$. However, blood reaching all regions of the parabronchus is at the same high $\mathrm{PCO}_{2}$ level. The $\mathrm{PO}_{2}$ levels of the blood that exits the lung is thus determined by the admixture of blood from all of the capillaries along each parabronchus, a design that makes it possible for the blood leaving the exchanger to have 

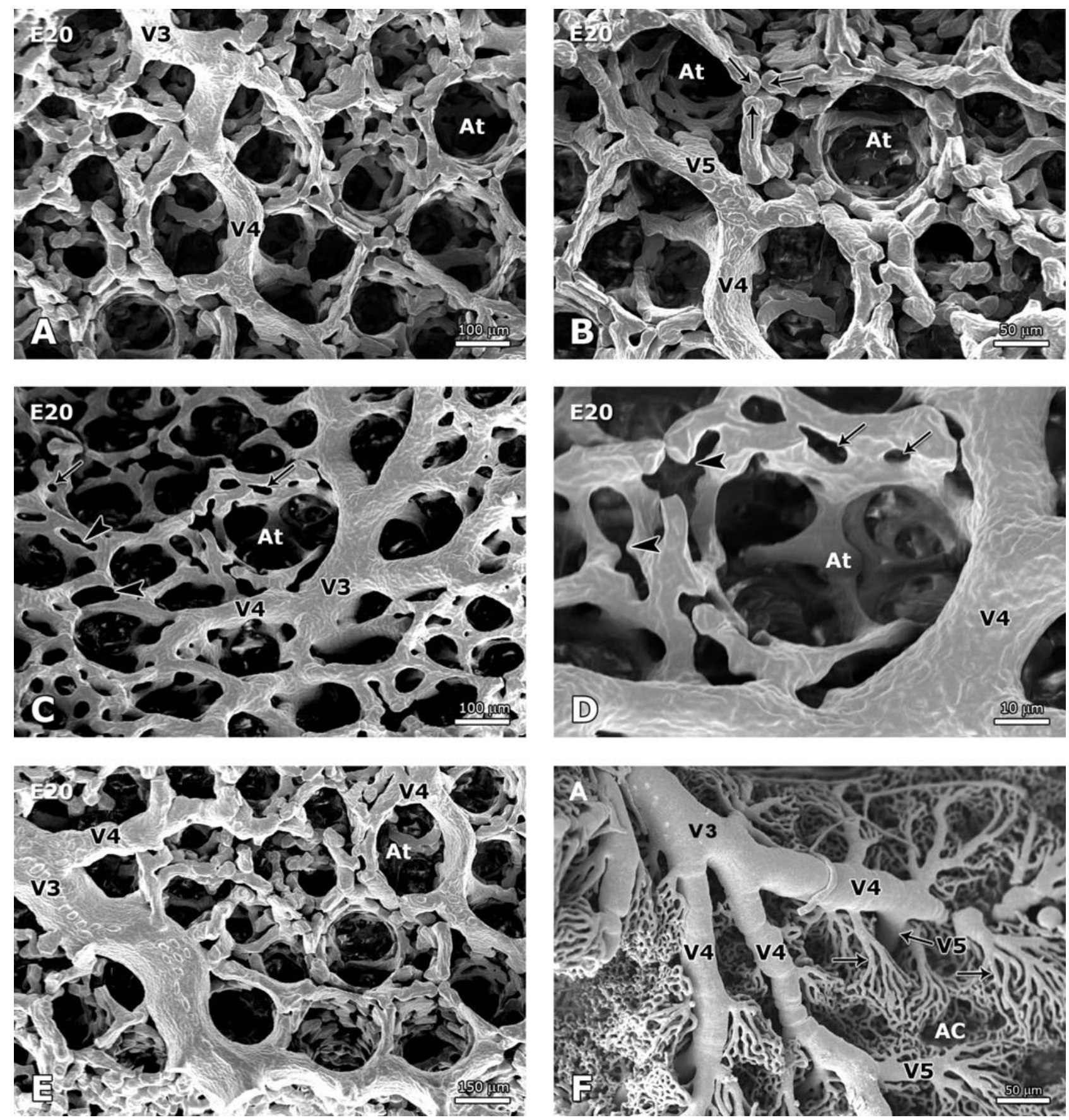

Fig. 4. Scanning electron micrographs of intravascular corrosion casts showing the establishment of the vascular networks around the At and ACs on the internal aspect of the PB at early E20 ( $A$ and $B)$ and at late E20 $(C$ and $D)$. The abbreviations for veins are V3 for intraparabronchial vein, V4 for atrial vein, and V5 for septal venule. $A$ and $B$ : the initial vascular plexus is established by sprouting and anastomoses around the already established air conduits. Subsequent migration and fusion delineate the draining vessels, such as the septal venule (V5), the atrial vein (V4), and the intraparabronchial vein (V3). Notice the spaces representing the At clearly outlined by the incipient vessels (arrows). $C$ and $D$ : remodeling of the basic pattern of the vasculature proceeds through intussusceptive angiogenesis, a process that entails formation of tissue pillars, which divide the vessel lumens. Such pillars are represented by holes in vascular casts (arrows), and their subsequent fusion (arrowheads) results in smaller branches around the At. Note the already defined draining vessels: the atrial vein (V4) and the intraparabronchial vein (V3). $E$ and $F$ : various generations of draining vessels are clearly defined as early as E20 and in the mature lung the pattern is maintained. The septal veins (V5 in $F$ ) are closely associated with the atria, run in the interatrial septa and confluence to form the atrial veins (V4), which drain into intraparabronchial veins (V3). Notice the space occupied by the atrium in the developing lung (At in $E$ ) and the smooth nature of the capillaries surrounding the air capillary (AC) in the adult lung $(F)$

a higher $\mathrm{Po}_{2}$ than that in the gas leaving the exchanger $(10,29$, 31). The arrangement of the parabronchial arteries at the periphery is such that small, successive regions of the parabronhus are supplied with deoxygenated blood of uniform composition, and such regions are drained by intraparabronchial veins. Such an arrangement has been described as a multicapillary serial arterialization and is responsible for the cross-current behavior (19). Here we have demonstrated that the relationship between air flow 

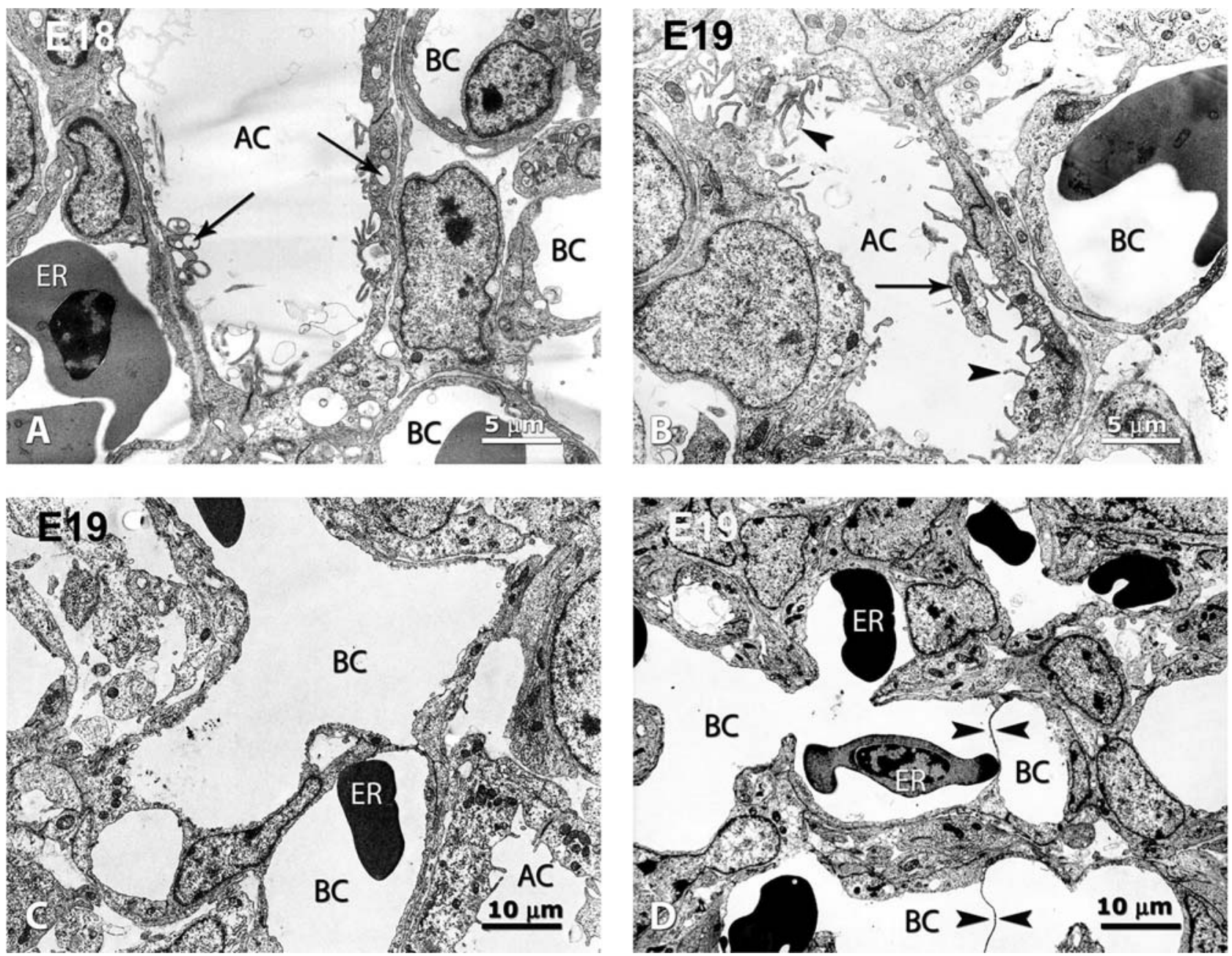

Fig. 5. Transmission electron micrographs showing the interaction between ACs and BCs during establishment of the blood-gas barrier ( $A$ and $B)$, as well as the fusion of the BCs $(C$ and $D)$. $A$ and $B$ : the thinning of the AC epithelium involves complex processes of cell cutting, which include vesiculation (arrows in $A$ ) and vacuolation and severing of the apical parts of cells (arrows in B). At this stage, ACs are distinguished from BCs due to the presence of microplicae (arrowheads in $B$ ) that result from vesicular rapture. In contrast, BCs are smooth and contain erythrocytes (ERs). $C$ and $D$ : mechanisms of capillary fusion are poorly understood, but, as evident from the transmission electron microscopy micrographs here, apposition of anastomosing walls of adjacent capillaries (BC in $C$, arrowheads in $D$ ) results in progressive thinning, perhaps due to hemodynamic pressure, until communication between the adjacent capillaries is established. Notice ERs apposed to the thinning wall.

in the air capillaries and blood flow in the gas exchange capillaries, where actual gas exchange occurs, is cross-current.

Previous descriptions of the cross-current system did not take into account the various generations of the blood vessels associated with the parabronchus, a fact that has been elucidated in the present study (Table 2). Indeed, the orientation of the parabronchial capillaries in relation to the bulk unidirectional gas flow in the parabronchial lumen is multidirectional (see Fig. $1 G$ ), where exchange capillaries converge to form the small venules and veins that ultimately gather to form the intraparabronchial veins. The latter veins drain large portions of the parabronchus. This arrangement ensures that consecutive regions of the parabronchus are exposed to new deoxygenated blood, therefore helping to maintain the concentration gradient at a reasonably high level. Nevertheless, the partial pressure of oxygen $\left(\mathrm{PO}_{2}\right)$ in the parabronchial lumen decreases distally, while the $\mathrm{PCO}_{2}$ increases (30). Descriptions of the direction of blood flow in other generations of the blood vessels presented here elucidate their respective 3D arrange- ment and have no direct implication on the cross-current flow. The bulk of the secondary bronchi are gas-exchanging conduits and behave much like the parabronchi, with the exception of the first medioventral secondary bronchus and the small initial parts of the rest of the secondary bronchi (22).

The avian cross-current gas exchange system is more efficient than the mammalian uniform pool system in that $\mathrm{PO}_{2}$ of blood leaving the parabronchi can exceed that in the gas exiting the parabronchi (10). In the uniform pool system, equilibration of the $\mathrm{PO}_{2}$ occurs. The countercurrent system of fish is the most efficient among vertebrates (35), but requires that there be a different entry and exit route for the oxygen-transmitting medium (water), a design not accomplished in birds. Our observations in the present study are that, early in development, the parabronchial capillaries form a disorganized meshwork, which is remodeled toward hatching, to assume the orthogonal disposition, with respect to the air capillaries.

The intricate arrangements of the blood vessels and air conduits in the avian lung lend mechanical strength to one 


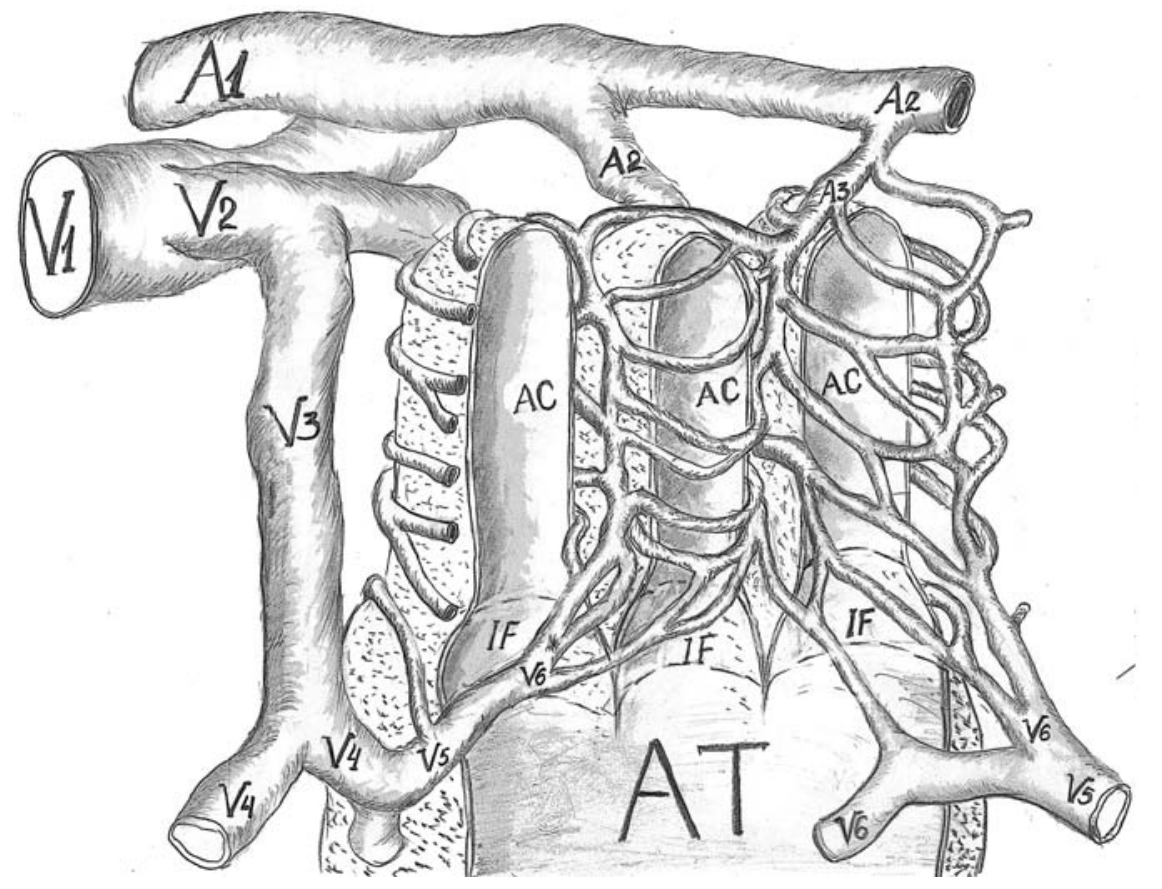

Fig. 6. A schema showing the interaction of the BCs with the ACs in the adult chicken. In the interparabronchial septum are the large supplying arteries (A1, A2, and A3) and draining veins (V1 and V2). The smallest draining vessels (V4, V5, and V6) are located on the interior aspect of the PB. They converge to form V3, which traverses the gas exchange mantle to join the large draining vessels. The names of the vessels are as provided above and detailed in Table 1. AT, atrium; IF, infundibulum.

another (32), and, by so doing, the avian lung is able to dispense with the bulk of interstitial tissue, thus allowing enormous space for gas exchange while still remaining structurally strong and noncompliant. The venous and arterial systems were identifiable in the intravascular casts, according to their topographical locations with respect to the parabronchial lumen (Fig. 1), and confirmed in semithin sections (see Fig. 2). In the latter situation, arteries had relatively thicker walls and more circular profiles compared with their cognate veins. During development, the abundant interparabronchial mesenchymal tissue was progressively reduced into a thin septum containing the interparabronchial vasculature, while the parabronchial mantle comprising interlacing blood and air capillaries was established.

The ontogeny of the avian pulmonary vasculature has received some attention from contemporary investigators, with the notion that, in development, it initiates by vasculogenesis $(3,16)$ and that expansion, patterning, and remodeling are through sprouting and intussusceptive angiogenesis (23). The process of intussusceptive angiogenesis was first reported by Caduff et al. (5) and is now well documented [see reviews by
Burri et al. (4), Djonov and Makanya (7), Makanya et al. (24)]. The quintessence of intussusceptive angiogenesis in intravascular casts is the presence of pillar holes, a feature characteristic in developing subjects and virtually absent in the adults (see, for example, Fig. 4).

Unequivocal delineation of the arterial and venous systems in the present study enables investigation into the preponderant angiogenic mechanisms and their spatiotemporal occurrence in the arterial and venous systems. In the arterial system, opposing parabronchial arterioles approach each other on the external surface of the parabronchus, appose, and fuse to form evanescent arcades that surround the parabronchi. Such arcades are soon broken down by intussusceptive branching remodeling to form parabronchial capillaries. On the basis of size and location, it is possible to delineate the arteries into three generations: the first one (A1) being the large interparabronchial arteries dominating the interparabronchial septa and running parallel to the long axes of the parabronchi. Parabronchial arteries (A2) branch from, and are orthogonal to, the A1 and give rise to $\mathrm{A} 3$, the arterioles that feed the parabronchial capillary meshwork. Sometimes A3 form filial branches, which

Table 2. Vessel generation, name, blood flow direction, and blood oxygenation status in the various categories of blood vessels encountered at the parabronchial unit

\begin{tabular}{|c|c|c|c|c|}
\hline Vessel Generation & Vessel Name & Flow Direction & Related Air Conduits & Blood Oxygenation Status \\
\hline A1 & Interparabronchial artery & Cocurrent & Parabronchi & Deoxygenated \\
\hline A2 & Parabronchial artery & Cross-current & Parabronchi & Deoxygenated \\
\hline \multirow[t]{2}{*}{ A3 } & Parabronchial arteriole & Cross-current & Parabronchi & Deoxygenated \\
\hline & Parabronchial capillaries & Cross-current & Air capillaries & Oxygenation occurs \\
\hline V6 & Infundibular venule & Cross-current Countercurrent; cocurrent & Infundibulae, parabronchi & Oxygenated \\
\hline V5 & Septal venule & Cross-current, Countercurrent; cocurrent & Atria, parabronchi & Oxygenated \\
\hline V4 & Atrial vein & Cross-current, Countercurrent; cocurrent & Atria, parabronchi & Oxygenated \\
\hline V3 & Intraparabronchial vein & Cross-current & Parabronchi & Oxygenated \\
\hline $\mathrm{V} 2$ & Parabronchial vein & Cross-current & Parabronchi & Oxygenated \\
\hline V1 & Interparabronchial vein & Countercurrent & Parabronchi & Oxygenated \\
\hline
\end{tabular}

$\mathrm{A}$, artery; V, vein. 
are broken down into gas exchange capillaries of the parabronchial mantle.

On the internal aspect of the parabronchi, the convergence of capillaries forms the infundibular venules, denoted as vessel generation 6 (V6) associated with individual infundibulae, as well as septal venules (V5) running in the interatrial septa. The septal venules may converge to form the atrial veins (V4), or sometimes join the intraparabronchial veins (V3) directly. Each atrial vein drains a group of approximately four atria, what is referred to by West et al. (34) as a microvascular unit. Anastomoses of large vascular sprouts initially establish large and irregular vascular entities around the atria (see Fig. 4), which are then broken down to small venules and fine capillaries surrounding the atria and air capillaries, respectively, mainly by intussusceptive remodeling. The capillaries grow toward the parabronchial mantle, where they anastomose with their cognates from the arterial system, thus participating in the establishment of the complex gas exchange tissue. The precise mechanisms and the chronology of events that entail the establishment of the discrete arterial and venous systems and the relationship between the two during development are unclear.

The ultimate target of the air capillaries and blood capillaries is to establish the thin blood-gas barrier typical of the avian lung. Indeed, the thinnest blood-gas barrier ever measured is encountered in the rock martin (Ptyonoprogne fuligula), estimated at only $0.09 \mu \mathrm{m}(21)$, rarely exceeds $0.5 \mu \mathrm{m}$ in other birds, and is exceeded by that in mammals by $>50 \%$ (20). Establishment of the thin blood-gas barrier in mammals is known to proceed through cell elongation, extrusion of lamellar bodies, and thinning (26). In chickens, the process is more complex and entails vesiculation, vacuolation, and even double membrane formation in a process referred to as secarecytosis or through cell constriction and pinching off (peremerecytosis) (25).

Mechanisms of capillary fusion are poorly understood, but, plausibly, for any network of vessels to be accomplished, capillary anatomoses have to occur. In the present study, developing capillaries appeared to abutt end to end, and this was followed by progressive thinning of the apposing regions, probably due to the hemodynamic pressure within the capillary lumen. Indeed, hemodynamic forces have been shown to play a role in vascular tree establishment during angiogenesis (6).

Although both intussusceptive angiogenesis and secarecytosis have been implicated in the establishment of the 3D arrangement of the blood and air capillaries, respectively, molecular control of these two processes is unclear. Intussusceptive angiogenesis has been documented since about 2 decades ago, and, over the years, overwhelming details regarding its inauguration, progression, and accomplishment have been documented (24). In contrast, secarecytosis has only recently been described (25). Further investigations to show the molecular control of these two processes, as well those governing mechanisms that lead to capillary fusions, are recommended.

\section{ACKNOWLEDGMENTS}

We thank, Krystyna Sala, Barbara Krieger, Christoph Lehmann, and Clemens Weber for excellent technical assistance. We are grateful to the three anonymous reviewers whose comments greatly helped in improvement of the quality of this article.

\section{GRANTS}

We acknowledge financial support from the Swiss National Science Foundation through Grants 31-45831.95, 31-55895.98, and 31-55895.98/2.

\section{REFERENCES}

1. Abdalla MA, King AS. The functional anatomy of the pulmonary circulation of the domestic fowl. Respir Physiol 23: 267-290, 1975.

2. Abdalla MA, King AS. The avian bronchial arteries: species variations. $J$ Anat 123: 697-704, 1977.

3. Anderson-Berry A, O'Brien EA, Bleyl SB, Lawson A, Gundersen N, Ryssman D, Sweeley J, Dahl MJ, Drake CJ, Schoenwolf GC, Albertine KH. Vasculogenesis drives pulmonary vascular growth in the developing chick embryo. Dev Dyn 233: 145-153, 2005.

4. Burri PH, Hushchuk R, Djonov V. Intussusceptive angiogenesis: its emergence, its characteristics, and its significance. Dev Dyn 231: 474-488, 2004.

5. Caduff JH, Fischer LC, Burri PH. Scanning electron microscope study of the developing microvasculature in the postnatal rat lung. Anat Rec 216: 154-164, 1986.

6. Djonov V, Baum O, Burri PH. Vascular remodeling by intussusceptive angiogenesis. Cell Tissue Res 314: 107-117, 2003.

7. Djonov V, Makanya AN. New insights into intussusceptive angiogenesis. EXS 94: 17-33, 2005.

8. Duncker HR. The lung air sac system of birds. A contribution to the functional anatomy of the respiratory apparatus. Ergeb Anat Entwicklungsgesch 45: 7-171, 1971.

9. Farmer CG. On the origin of avian air sacs. Respir Physiol Neurobiol 154: 89-106, 2006.

10. Fedde MR. Relationship of structure and function of the avian respiratory system to disease susceptibility. Poult Sci 77: 1130-1138, 1998.

11. King AS, McLelland J. Birds: Their Structure and Function. London: Ballie're Tindall, 1984.

12. Locy WA, Larsell O. The embryology of the bird's lung based on observations of the domestic fowl: part II. Am J Anat 20: 1-44, 1916.

14. Maina JN. A scanning electron microscopic study of the air and blood capillaries of the lung of the domestic fowl (Gallus domesticus). Experientia 38: 614-616, 1982.

15. Maina JN. Scanning electron microscope study of the spatial organization of the air and blood conducting components of the avian lung (Gallus gallus variant domesticus). Anat Rec 222: 145-153, 1988.

16. Maina JN. Systematic analysis of hematopoietic, vasculogenetic, and angiogenetic phases in the developing embryonic avian lung, Gallus gallus variant domesticus. Tissue Cell 36: 307-322, 2004.

17. Maina JN. Spectacularly robust! Tensegrity principle explains the mechanical strength of the avian lung. Respir Physiol Neurobiol 155: 1-10, 2007.

18. Maina JN. A systematic study of the development of the airway (bronchial) system of the avian lung from days 3 to 26 of embryogenesis: a transmission electron microscopic study on the domestic fowl, Gallus gallus variant domesticus. Tissue Cell 35: 375-391, 2003.

19. Maina JN. Structure, function and evolution of the gas exchangers: comparative perspectives. J Anat 201: 281-304, 2002.

20. Maina JN. Development, structure, and function of a novel respiratory organ, the lung-air sac system of birds: to go where no other vertebrate has gone. Biol Rev Camb Philos Soc 81: 545-579, 2006.

21. Maina JN. Morphometrics of the avian lung. 3. The structural design of the passerine lung. Respir Physiol 55: 291-307, 1984.

22. Makanya AN, Djonov V. Development and spatial organization of the air conduits in the lung of the domestic fowl, Gallus gallus variant domesticus. Microsc Res Tech 71: 689-702, 2008.

23. Makanya AN, Hlushchuk R, Baum O, Velinov N, Ochs M, Djonov V. Microvascular endowment in the developing chicken embryo lung. Am J Physiol Lung Cell Mol Physiol 292: L1136-L1146, 2007.

24. Makanya AN, Hlushchuk R, Djonov V. Intussusceptive angiogenesis and its role in vascular morphogenesis, patterning and remodeling. Angiogenesis; doi:10.100175/10456-009-9129-5.

25. Makanya AN, Hlushchuk R, Duncker HR, Draeger A, Djonov V. Epithelial transformations in the establishment of the blood-gas barrier in the developing chick embryo lung. Dev Dyn 235: 68-81, 2006.

26. Makanya AN, Sparrow MP, Warui CN, Mwangi DK, Burri PH. Morphological analysis of the postnatally developing marsupial lung: the quokka wallaby. Anat Rec 262: 253-265, 2001.

27. Makanya AN, Stauffer D, Ribatti D, Burri PH, Djonov V. Microvascular growth, development, and remodeling in the embryonic avian kid- 
ney: the interplay between sprouting and intussusceptive angiogenic mechanisms. Microsc Res Tech 66: 275-288, 2005.

28. Nasu T. Scanning electron microscopic study on the microarchitecture of the vascular system in the pigeon lung. J Vet Med Sci 67: 1071-1074, 2005.

29. Piiper J, Scheid P. Models for a comparative functional analysis of gas exchange organs in vertebrates. J Appl Physiol 53: 1321-1329, 1982.

30. Scheid P. Respiration and control of breathing. In: Avian Biology. New York: Academic, 1982, p. 405-453.

31. Scheid P, Piiper J. Cross-current gas exchange in avian lungs: effects of reversed parabronchial air flow in ducks. Respir Physiol 16: 304-312, 1972.
32. Watson RR, Fu Z, West JB. Minimal distensibility of pulmonary capillaries in avian lungs compared with mammalian lungs. Respir Physiol Neurobiol 160: 208-214, 2008.

33. West JB, Watson RR, Fu Z. The honeycomb-like structure of the bird lung allows a uniquely thin blood-gas barrier. Respir Physiol Neurobiol 152: $115-118,2006$

34. West NH, Bamford OS, Jones DR. A scanning electron microscope study of the microvasculature of the avian lung. Cell Tissue Res 176: 553-564, 1977.

35. Wilson JM, Laurent P. Fish gill morphology: inside out. J Exp Zool 293: 192-213, 2002. 